\title{
Le matematiche pure e miste nei primi dodici Congressi della Società Italiana per il pro- gresso delle scienze ${ }^{(*)}$.
}

\author{
(Di Valentino Cerruti, a Roma.)
}

\section{ONOREVOLI COLLEGHI,}

Prima di dar principio a' nostri lavori consentitemi uno sguardo retrospettivo all'opera, che la Società Italiana per il progresso delle scienze, risorgente ora a vita novella, ha saputo compiere, in pochi ed omai lontani anni di esistenza, nel campo speciale degli studi matematici. Restringerò il mio discorso quasi esclusivamente alle sessioni tenute dalla Società dal 1839 al 1847, durante il quale periodo essa ha spiegato un'azione davvero feconda e che per molti capi si potrebbe, senza esagerazione, qualificare come eroica. Nelle tre sessioni posteriori al 1847, sessioni rinnovate dopo non breve sosta e seguite ad intervalli saltuari, relativamente esigue furono le manifestazioni in ogni ramo dell'umano sapere, ed anche le matematiche non somministrarono materia a comunicazioni o a discussioni che valgano a richiamare sopra di sè particolare attenzione. Ad esumare, in forma sia pure sommaria, la storia del passato, mi muove un sentimento di gratitudine, sempre doverosa e più oggi, che ci proponiamo di rinverdire una istituzione tanto benemerita del pensiero nazionale: un sentimento di gratitudine verso la memoria di uomini preclari, che onorarono il paese colla forza dell'ingegno, e non esitarono di sacrificare, quando fu necessario, la quiete e gli ideali dello scienziato per correre i rischi di creare una patria e sobbarcarsi alle oscure e rudi fatiche di ordinare lo Stato. De' quali è bello a noi, venuti per

(*) Discorso pronunciato in Parma il dì 24 settembre 1907 inaugurandosi i lavori della Sezione I della Societả Italiana per il progresso delle Scienze. (Prima riunione della Societì, Parma, settembre 1907). 
virtù loro in tempi favorevoli al culto pacifico degli studi, salvare dall'oblio i pensamenti e custodire con venerazione il patrimonio scientifico, che ci han legato, senza guardare se sia modesto e non quale l'avrebbe desiderato la nostra ambizione patriottica.

Pretro Paoli, dotto professore di Analisi nell'Ateneo pisano, descriveva a foschi colori, nella prefazione al suo trattato di algebra $\left({ }^{1}\right)$, pubblicato nei primi anni del secolo scorso, le sorti miserande dell'insegnamento delle matematiche nel nostro paese. Le parole del PAOLI, concesso che rispondessero al vero in senso assoluto, sarebbe stato ingiusto applicarle in quel momento all'Italia sola, imperocchè dappertutto, anche fuori d'Italia, ove si eccettui Parigi, le scuole di matematica non erano molte diverse o migliori di quel che fossero presso di noi. Certamente un insegnamento fiacco ed angusto non era fatto per promuovere il progresso degli alti studi: ma l'industria individuale sopperiva alle manchevolezze delle pubbliche istituzioni. Onde in quel tempo l'Italia poteva giustamente gloriarsi de' nomi di LaGRange, Malfatri, Ruffini, Brunacai, Ortani, Nigola Fergola, e dello stesso Paoli, per non citare che $\mathrm{i}$ nomi maggiori e più noti, mentre si era appena chiusa la tomba del Mascheroni, rapito immaturamente in ancor fresca età alle scienze ed alle lettere.

Le censure del Paoli, ripetute più tardi e in diverse occasioni con frasi troppo recise e senza le opportune riserve da uomini di grande autorità $\left({ }^{2}\right)$, generarono il pregiudizio che la produzione italiana nelle matematiche pure e miste della prima metà del secolo XIX abbia così tenue importanza da non meritare di essere considerata nella storia del movimento generale delle scienze dopo le grandi commozioni politiche e militari dell'epoca napoleonica. Parmi invece che ove la si esamini con diligenza e senza passione o prevenzioni, lasciando pure in disparte ogni velleità di confronto, il quale riuscirebbe troppo sproporzionato, colle immortali creazioni contemporanee de' grandi matematici della Francia e della Germania, da nessun equo estimatore si vorrà negare un posto onorevole ai geometri italiani, ai quali più che il valore è mancata la fortuna. A suffragio di questa opinione sarebbe facile ricavare argomenti decisivi dallo spoglio di atti accademici e di raccolte scientifiche, che si venivano stampando in varie città d'Italia, raccolte scientifiche in gran parte oggidì pressochè ignorate dall'universale, dagli eru- 
diti in fuori. Ma io non intendo di uscire dal compito che mi sono imposto, di considerare unicamente i lavori prodotti ne' Congressi della nostra Società, o che direttamente vi si collegano, tanto più che mi paiono bastevoli da sè a convincere chicchessia della verità della mia affermazione.

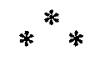

E vengo senza più al proposito mio cominciando dalle matematiche pure: nelle quali ci si presenta in prima linea il nome altamente stimabile. del professore Féice Ghò̀. Il Chiò esordiva nella carriera scientifica comunicando al Congresso di Torino (1840) un notevole teorema sulla convergenza delle serie trigonometriche, teorema che fece molto onore al MaLmsten $\left(^{3}\right.$, il quale lo ridiscoverse qualche anno dopo, e che ora suole essere riprodotto ne' trattati di analisi colla dimostrazione datane da H. Holmogren ${ }^{4}$ ).

Nuovo e più apprezzato saggio del suo ingegno forniva il CHrò nel Congresso di Genova (1846) proponendo due formule relative alla trasformazione di una funzione arbitraria in un integrale definito doppio dello stesso genere di quella di Fourier, formule che egli poi utilizzava nella determinazione di qualche integrale definito conosciuto e di qualche altro nuovo. In quel medesimo Congresso poneva il suggello alla fama di abile analista rendendo conto delle sue classiche ricerche intorno alla serie di LaGRange, iniziate allo scopo di rettificare una conclusione fallace contenuta nella Nota XI del Traité de la résolution des équations numériques de tous les degrés del sommo geometra torinese. Le quali ricerche poi egli ridusse in tre Memorie: le prime due inserite, con relazioni lusinghiere del Gavchy, tra quelle dell'Accademia delle Scienze di Parigi e la terza pubblicata, dopo la morte ed in compendio, negli Atti dell'Accademia delle scienze di Torino ( $\left.{ }^{5}\right)$.

Antagonista del Chiò sorse a Genova il Menabrea $\left(^{6}\right)$, propugnatore convinto, ma non egualmente felice, della teoria lagrangiana, alla cui illustrazione dedicò in quel torno di tempo, due estesi lavori e poi, trascorsi trent'anni, sparito già il CHò, vari articoli di polemica col Genocchr ( ${ }^{7}$ ).

Uno dei teoremi fondamentali scoverti dal CHIò intorno a una condizione, la quale, quando è verificata, assicura la convergenza della serie, fu in seguito ridimostrata con procedimento più semplice e più intuitivo da altri, ma le disquisizioni minute nelle quali egli si è addentrato circa la ricognizione dell'effettivo campo di convergenza e l'applicazione della serie alla risoluzione delle equazioni, nonchè le dispute vivaci che ne consegui- 
rono, potrebbero oggi ancora essere studiate con frutto e provocare nuove $\mathrm{e}$ interessanti indagini.

Tra i collaboratori più attivi e costanti de'vari Congressi va collocato Serafrno Munich, professore di calcolo infinitesimale nell'Università di Padova. Le sue comunicazioni si aggirano su multiformi soggetti: la geometria cinematica, l'eliminazione delle funzioni arbitrarie, l'integrazione delle equazioni alle derivate parziali, le condizioni d'integrabilità delle forme differenziali ed alle differenze finite, dove sono ampliati alcuni teoremi di LAGRANGE e di LisRr, ed altre intorno ai trascendenti ellittici ed abeliani. Delle quali ultime, esposte al Congresso di Venezia (1847), non si hanno che i titoli desunti dal diario del Congresso, mancando gli Atti che non furono pubblicati, ma se ne può arguire il contenuto dalle Memorie sullo stesso sog. getto, che il Minich presentò in quel medesimo anno all'Istituto veneto. Tra esse piacemi segnalare la Memoria sulla integrazione algebrica di un sistema di equazioni differenziali iperellittiche $\left(^{8}\right)$. La integrazione algebrica di tal sistema di equazioni, così per via indiretta come per via diretta, fu oggetto di iterate ricerche da parte di JACOBI, Richelot, HaEDEnkamp, Lrouville e più tardi anche del Genocan e del Brioschi $\left(^{9}\right)$. Il lavoro del Mrnich contiene un nuovo procedimento di integrazione diretta e tra i vari lavori ora ricordati, che lo hanno preceduto o seguito, tiene sempre un posto ragguardevole.

Rilevo ancora dal diario testè ricordato, che il MINIcH prestò viva attenzione ad uno studio sulla moltiplicazione e la divisione de' trascendenti abeliani di prima specie, svolto avanti al Congresso dal dott. Gurdo Susanr. Non sono riuscito a scoprire se e dove il Susañ abbia pubblicato il suo lavoro; malgrado ciò parvemi conveniente non dimenticarlo in questa rassegna, come dimostrazione che in quei tempi non mancava tra noi chi si occupasse delle teorie più astruse dell'analisi matematica. Fatto tanto più notabile nel caso particolare, ove si pensi che le celebri ricerche dell'Hermite sul medesimo soggetto risalivano a soli tre o quattro anni innanzi, e nel 1847 non avevano riceruto che una diffusione assai limitata.

In un dominio affine, cioè nel dominio delle trascendenti ellittiche e più propriamente delle loro applicazioni alla geometria, rientra la parte meglio conosciuta del contributo recato dal prof. Nicola Trudi al Congresso di Napoli (1845). JACOBI, invocando il soccorso delle funzioni ellittiche, aveva dedicato una elegante Memoria allo studio delle proprieta dei poligoni di PoNCELET, cioè de'poligoni simultaneamente inscritti in una sezione conica e circoscritti ad una seconda, nel caso speciale in cui le due coniche si ridu- 
cono a due cerchi interni l'uno all'altro, donde in particolare scaturiva una costruzione delle formule per la moltiplicazione. Il Trudi completò lo studio di JACoBI, estendendolo al caso di due coniche qualunque ed in una giacitura rispettiva assolutamente generale, mediante un singolar processo di eliminazione, che, a quanto è asserito negli Atti del Congresso, sembra aver condotto l'autore ad altri pregevoli risultati nella dottrina delle equazioni binomie e nell'assegnazione delle condizioni per la risolubilità algebrica di equazioni di grado superiore al quarto. Checchè sia di queste ultime indicazioni sulle quali non seppi mettere insieme notizie concrete, riguardo alla prima parte dell'opera del Trudr si hanno elementi sicuri di giudizio in una serie di Memorie, che egli più tardi e a varie riprese consegnò negli Atti dell'Accademia delle scienze di Napoli; Memorie che per profondità e generalità di ricerca in nulla cedono ai lavori quasi contemporanei del CAYLEY, del SALMON e del Brioschi e forse li superano in perspicuità e genialità di esposizione $\left(^{10}\right)$.

In una direzione totalmente diversa si distinsero Paolo Frisians, astronomo nella Specola di Brera, e Giovannr Maria Lavagna, professore nell'Università di Pisa. Del primo è negli Atti del Congresso di Milano (1844) registrata un'utile monografia sulle equazioni trascendenti e nel Congresso di Venezia (1847) un lavoro di maggior polso sul problema di PFAFF, pubblicati poi entrambi per disteso nelle Effemeridi astronomiche di Milano $\left({ }^{11}\right)$. Questa cicostanza salvò le due produzioni del Frisiani da un completo oblio: invece sorte più disgraziata attendeva la Memoria letta dal LAFAGNA nel Congresso di Napoli (1845) sull'integrazione delle equazioni non lineari alle derivate parziali del prim'ordine con un número qualunque di variabili. Sebbene sia negli Atti del Congresso un'analisi abbastanza larga della Memoria, che permette di ricomporla nelle sue linee essenziali, e nei volumi dell'Accademia Gioenia di Catania per l'anno 1850 il lavoro completo quale fu esibito al Congresso $\left({ }^{12}\right)$, essa passò inosservata, ed a torto. Perchè si tratta di uno studio eccellente, nel quale con procedimento alquanto diverso da quello proposto da JACOBI, è ampliato al caso di un numero qualunque di variabili il metodo d'integrazione escogitato dal Lagravge pel caso di due variabili indipendenti. Ed anche oggi può riuscire non senza vantaggio conoscere gli espedienti a cui ricorre l'autore per costruire l'integrale generale e per risolvere il problema di Gauchy.

Giunto a questo punto il mio pensiero si volge spontaneo verso il decano de' nostri matematici, uno de' pochissimi superstiti fra quanti parteciparono operosamente ai Congressi anteriori alle guerre per l'indipendenza 
nazionale, al prof. TARDY, che dopo lunghi anni consacrati all'insegnamento, passa ora quasi nonagenario l'estrema vecchiezza in onorato riposo. Presentò il Tardy al Congresso di Milano (1844) una Memoria sui differenziali a indice fratto; essa fu sottoposta all'esame di una Commissione formata del Prola e del Plana ed ebbe favorevole il voto de' due illustri personaggi. Non mi risulta che la Memoria sia stata divulgata per le stampe; ciò non di meno se ne può ricavare la sostanza dal rapporto del Prola e del Plana che figura negli Atti del Congresso e dal testo di un'altra Memoria col medesimo titolo venuta alla luce quattordici anni dopo, nel primo tomo degli Annali di Matematica pura ed applicata $\left({ }^{13}\right)$; dove è da notare la definizione di derivata secondo un indice qualunque dedotta come ovvia e naturale estensione da quella che dette il LaPLAce per le derivate ordinarie col mezzo di un integrale definito. Il quale integrale a sua volta non differisce dall'integrale ben noto del ${ }^{4} \mathrm{CAUCH}_{\mathrm{U}}$ che esprime il valore di una funzione uniforme di variabile complessa o di una sua qualsivoglia derivata ordinaria nel centro di un cerchio, quando sia conosciuta la successione de'valori della funzione al contorno. Tale definizione non collima colla definizione a cui si era fermato il Lrouville in numerose pubblicazioni sul medesimo argomento e le è di gran lunga preferibile. Dei procedimenti spiegati nel suo lavoro fece il TARDY uso sistematico in una Memoria di squisita fattura sul moto dell'acqua in vasi di varia forma, stampata separatamente in Firenze nel $1847\left({ }^{14}\right)$, che fu poi oggetto di varie acute osservazioni da parte del Genocchi $\left({ }^{15}\right)$.

Coronamento della prima parte di questa mia cursoria enumerazione sarà il ricordo, per noi incancellabile, dell'intervento di BorchardT e di JacoBr al Congresso di Lucca (1843). Borchardt vi lesse una Memoria sopra certi sistemi di equazioni differenziali non lineari, che ammettono un numero limitato di integrali algebrici, conducenti ad alcune note formule per la trasformazione delle funzioni ellittiche $\left({ }^{16}\right)$. JACOBI vi enunciò un teorema relativo alla teoria dell'ultimo moltiplicatore il quale offre una regola semplice e certa per costruire, conosciuto che sia il moltipiicatore di un sistema di equazioni differenziali del prim'ordine, il moltiplicatore corrispondente per il sistema ridotto, che si trae dal dato, quando se ne possegga un integrale contenente una costante arbitraria.

La presenza di Bonchand e di Jacobi al Congresso di Lucca è memorabile altresì, perchè coincide con un momento importante nel rinnovamento degli studi matematici presso di noi. JACOBг aveva nel 1843 intrapreso per ragioni di salute, in compagnia di Steinen e di BorchaRDT, un viaggio in 
Italia e si era soffermato a lungo in Roma ed in Napoli. Dimorando a Roma vi arricchì il Giornale Arcadico di varie comunicazioni in lingua italiana sopra soggetti di analisi e di meccanica $\left({ }^{17}\right)$, coadiuvato nella loro composizione in una lingua che non gli era troppo famigliare, dal Chesins, il quale ad alcune aggiunse di suo dei notevoli commenti. Contemporaneamente lo STEINER scopriva la celebre superficie speciale di quart'ordine della proprietà caratteristica di essere incontrata secondo due sezioni coniche da ogni suo piano tangente e pubblicava nella medesima rivista una interessante Memoria sulle coniche inscritte in un triangolo o in un quadrilatero e sulle coniche circoscritte ad un triangolo $o$ ad un quadrigono $\left({ }^{18}\right)$.

Anche la stanza in Napoli non passò vana per la scienza, imperocchè dalle conversazioni ivi avute con Jacobi e con Sterner trasse il Padula materia e incitamento a'suoi lavori più belli versanti intorno alle singolarità delle curve algebriche piane $\left({ }^{19}\right)$.

Nelle matematiche applicate emerge sopra tutte le altre la veneranda figura di Ottaviano Fabrizio Mossotti, professore di meccanica celeste e di fisica matematica nell'Università di Pisa. La massima parte delle sue comunicazioni nei vari Congressi, traggono origine e trovano ragione in una Memoria, oggi assai rara, che egli aveva fatto stampare nel 1836 in Torino, sulle forze regolatrici dell'intima struttura dei corpi $\left({ }^{20}\right)$. Da pochi anni era sorta, per opera principalmente di Navier, Cauchr e Poisson, la teoria generale della elasticità riposante sulla ipotesi della composizione corpuscolare della materia e delle azioni a distanza. Ammessa l'azione a distanza, da un punto di vista astratto e meramente matematico non fa certo difficolta immaginare che essa proceda secondo una funzione a piacere della distanza medesima, e possa anche, come suppose il Boscovich, col variare di essa mutare natura più volte di seguito, diventando ora attrattiva, ora repulsiva. $\mathrm{Ma}$ quel che può rimanere indifferente al matematico, altrettanto non avviene al fisico, il quale ama ricostruire i fatti multiformi e complessi della natura con elementi semplici e parlanti alla fantasia. Appunto per soddisfare ad un simile bisogno il Mossotr compose come una specie di modello, dal quale $\mathrm{i}$ fatti che nel suo tempo più tormentavano la mente dei fisici, ricevono, qualitativamente almeno, una spiegazione, o meglio una interpretazione semplice e rigorosa. Esclusa ogni azione elementare che non fosse 
un'attrazione od una repulsione newtoniana, immaginò tutto lo spazio riempito di un fluido od etere, le cui particelle si respingano tra loro colla legge suddetta, ma per tutto il resto goda delle proprietà dei fluidi ordinari. In questo fluido pensò disseminate le molecole ponderabili repulsive tra loro, ma attraenti le particelle dell'etere sempre colla legge newtoniana. In forza di considerazioni analoghe a quelle di cui fa uso LAPLAGE nel libro XII della Meccanica celeste, la pressione del fluido etereo dovrebbe risultare in ogni luogo proporzionale al quadrato della densità; posto questo il MossoTTr con sottile analisi pervenne a dimostrare, che l'etere in equilibrio si aggruppa intorno ad ogni molecola formando un'atmosfera di una densità grandissima nella contiguità della molecola, ma decrescente con tanta rapidità, che ad una distanza sensibile la condensazione può considerarsi come nulla e la densità confondersi con quella generale dell'etere nello spazio. Pertanto nel modello del Mossotтr i corpi ponderabili vanno concepiti come un aggregato di nuclei solidi "arnati di atmosfere eteree. Un calcolo elegante e condotto con singolare maestria lo condusse a riconoscere che l'azione reciproca di cosi fatti elementi segue tal legge da riuscire repulsiva e rapidamente decrescente nelle distanze minime, attrattiva nelle maggiori e conforme alla newtoniana nelle sensibili. Le idee del Mossotri sulla costituzione della materia furono nel decennio dal 1840 al 1850 vivamente discusse anche fuori d'Italia, massime in Inghilterra, e fornirono argomento a replicate dispute nei Congressi della nostra Società. Alle medesime idee sono informate le sue auree Lezioni di Fisica matematica $\left({ }^{21}\right)$.

Una delle applicazioni più notevoli di tali vedute fu certamente quella che egli fece nel Congresso di Firenze (1841) alla teoria della dispersione della luce. Osservando che nell'etere libero la dispersione non esiste o è inapprezzabile, il Mossortr suppone che la presenza delle molecole ponderabili generi, nello spazio da esso occupato, un'alterazione periodica nella densità e nella forza elastica dell'etere medesimo; e giunge così per la velocità di propagazione di un'onda di data lunghezza ad una formula la quale coincide colla formula dedotta dal CaUchr in ben diversa ipotesi. Un modo di vedere non dissimile fu accolto, ma altrimenti sviluppato, dal Briot ventitre anni appresso nei suoi saggi sulla teoria matematica della luce.

Un'altra applicazione descrisse il Mossotтr nel Congresso di Torino (1840), destinata a spiegare la generazione della forza contrattile superficiale introdotta dal Youna nella sua teoria dei fenomeni capillari, della qual teoria propose inoltre una semplice e chiara esposizione. Ad essa aggiunse poi nuove e più ampie dilucidazioni nel Congresso di Genova (1846). 
Di una terza ingegnosa applicazione è traccia negli Atti del Congresso di Siena (1862), diretta a chiarire il meccanismo delle scariche elettriche fra le nubi temporalesche e dell'azione protettrice dei parafulmini.

Ma oltre le comunicazioni, che in maniera diretta o indiretta si riconnettono colla sua dottrina intorno alla genesi delle forze molecolari, gli Atti dei Congressi ne contengono altre ancora di non minore importanza, le quali hanno resistito meglio al tempo e ai progressi della scienza.

Ad esempio negli Atti del Congresso di Lucca (184:3) è parola di una Memoria del Mossoters, riportata poi nel primo tomo degli Annali delle Universitì toscane, sulle proprietà degli spettri formati dai reticoli in cui, fondandosi sulle osservazioni di FraunHofer, stabilisce una espressione per la lunghezza d'onda corrispondente ad una data linea dello spettro in funzione della sua distanza della linea centrale. Negli Atti del Congresso di Genova (1846) sono riassunti i risultati delle sue ricerche originali, e sempre in onore, intorno alla teoria matematica dei dielettrici conforme alle vedute di Faraday, ricerche pubblicate quasi subito per disteso nelle Memorie della Società Italiana dei XL: e negli Atti del Congresso di Napoli (1845) è riferita una sua breve Nota sopra una espressione del termine generale dell'equazione del centro. Ma di qualche altra comunicazione, come ad esempio sulla deduzione delle formule di Fresser per la doppia rifrazione dalle equazioni del moto dei corpi elastici (Milano, 1844), sull'analisi dello spettro solare e riflessioni sulle teorie dell'ottica (Napoli, 1845), non mi fu dato di rintracciare che poco più dei semplici titoli.

Gran parte dei lavori del Mossotrt $\left({ }^{22}\right)$, ammirabili per eleganza e sobrietà di dettato e per originalità di vedute, si trovano dispersi in collezioni di difficile accesso o poco conosciute : ben si provvederebbe alla sua fama e più ancora al progresso degli studi ripubblicandole in un corpo unico.

Accanto al Mossottr deve porsi il Plana. Questi nel Congresso di Milano (1844) espose la sostanza della sua grossa Memoria: Sulla distribuzione permanente dell'elettricità alla superficie di due sfere isolate, stampata poi tra quelle dell'Accademia delle scienze di Torino $\left({ }^{23}\right)$. La Memoria del Plana, sebbene ristretta ad uno speciale e celeberrimo problema, può per certi lati riguardarsi come un trattato di elettrostatica. Essa è un commento ad una Nemoria classica di Porsson, in cui il problema dell'induzione mutua di due sfere era stato ridotto alla risoluzione di una equazione funzionale. Ma lo formule, che il Porsson ne aveva ricavate per il calcolo della densitì elettrica, offrivano il fianco a varie obiezioni e parerano condurre a risultati il- 
lusori Oltre a vari miglioramenti di minor conto, il Plana perfezionò la teoria del Poissor per guisa da piegarla alle valutazioni numeriche e la corredò di tabelle diligentemente calcolate, tuttora in uso presso i fisici, the permettono un immediato riscontro fra $\mathrm{j}$ risultati della teoria e le misure sperimentali. Si posseggono al certo oggridì delle risoluzioni del problema più elaborate e piì intuitive; ed è stato dimostrato recentemente dal Darboux l'attinenza della risoluzione del Porsson con quella che si trae col metodo delle immagini $\left({ }^{24}\right)$; ma non saprei dire se rispetto alle calcolazioni numeriche le nuove soluzioni somministrino delle formole più comode di quelle del PlaANa o almeno ignoro se per questo riguardo sia mai stato eseguito un confronto accurato e decisivo.

Di fronte ai lavori del Mossote e del Plana tutti gli altri paionmi assumere figura secondaria, sebbene tra essi non manchino di quelli non indegni che se ne conservi memoria. In via di esempio sarebbe ingiusto dimenticare che il Bfuri, distinto professore di fisica nell'Università di Pavia, srolgeva nel Congresso di Padova (1842) delle considerazioni matematiche sopra alcuni fenomeni geologici e vi esponera il progetto di una serie di osservazioni sistematiche al fine di risolvere la controversa questione sulla fluidità interna della terra.

E non si saprebbe nemmeno passare sotto silenzio il nome di Gaвrio Proca, il quale nel Congresso di Milano (1844) proponeva una nuova analisi del problema del moto dell'acqua ne' canali, quantunque incerte ed imperfette ne siano le conclusioni. Fu il Prosd zelante propagatore de'metorli della meceanica analitica di LAgravar e della nuova meccanica molecolare di Cauchy e Porsson; e si conciliò la gratitudine degli studiosi con un esteso trattato sugli integrali definiti, con un'esposizione ordinata del calcolo dei residui del Gaughy e colla traduzione italiana, in comune col Frisiani, corredata di estesi commenti di una Memoria dello stesso Cadahy sul calcolo de' limiti $\left({ }^{25}\right)$. Egli si occupò con singolare pertinacia, cui piacerebbe fosse toccato migliore successo, di problemi idrodinamici ed in ispecie del moto dellacqua nei fiumi e nei canali regolati. II Broschr nel curare la stampa di una Memoria postuma del Prold intorno al moto dell'acqua negli alrei scoperti $\left({ }^{26}\right)$, premise di suo una minuta recensione delle ricerche anteriori del Prola stesso e degli altri matematici italiani della prima metà del secolo XIX sullidraulica razionale. Dalla recensione del Brioschi risulta chiaramente che esse son tutte inquinate da due rizi fondamentali: uno analitico ed uno fisico. Il vizio analitico, oltre la introduzione quasi costante di 
un'ipotesi restrittiva, che esclude la possibilità di moti vorticosi, consiste in una non esatta nè completa posizione del problema rispetto alle condizioni ai limiti. Le soluzioni faticosamente combinate sono sempre più o meno particolari e non hanno mai tutta la voluta generalità per adattarsi ad una forma arbitraria delle pareti: o per lo meno le condizioni alle pareti non sono prese subito in considerazione ne' calcoli per subordinarvi la soluzione che si vuol costruire. Ed anche quando la soluzione ha generalità sufficiente, essa è presentata in forma tale che la determinazione degli elementi arbitrari mediante le condizioni ai limiti riesce pressochè impraticabile. Il vizio fisico riguarda gli effetti dell'attrito interno e dell'attrito alle pareti sempre trascurati: cosa concedibile finchè si tratta dell'esito dell'acqua da fori praticati in un vaso di limitate dimensioni, ma non più nel moto per lunghi tubi, per canali o fiumi, dove essi hanno influenza preponderante. Le ricerche del Prola e de'suoi contemporanei hanno radice comune nelle soluzioni trovate dal VentuRour per il moto dell'acqua in un raso conico rotondo ad asse verticale $\left({ }^{27}\right)$ e dal Grutro $\left({ }^{28}\right)$ in un vaso generato dalla rotazione di una iperbole cubica intorno ad un suo asintoto: soluzioni particolarissime e starei per dire fortuite, cioè non dedotte con metodo certo e razionale. Primo invece a risolvere correttamente un problema di simil genere fu il BeтTr $\left(^{20}\right)$, che riusci a determinare in modo rigoroso l'efflusso dell'acqua da un vaso parallelepipedo con una fessura rettangolare scavata, parallelamente a due pareti opposte, nel fondo supposto orizzontale. Per debito di giustizia non si può tuttavia dissimulare che malgrado la imperfezione dei lavori del VENTuRol e suoi seguaci, aveva pur saputo il BrDone, con ragionamento ingegnosissimo, trarne il valore del coefficiente di contrazione per una vena sgorgante da una luce circolare scolpita in parete sottile $\left({ }^{30}\right)$.

Insieme col Proca giova mentovare per indagini di idraulica teorica $\mathrm{il}$ prof. Vincenzo Amar dell'Università di Pisa, autore di un trattato di meccanica, che ebbe a' suoi dì buona riputazione come contenente alcune utili e, per quel tempo, nuove osservazioni sull'applicazione del principio delle velocità virtuali a'corpi continui o quando si tenga ragione della resistenza. d'attrito e per qualche elegante deduzione relativa al moto dei liquidi. Dei (quali perfezionamenti, di carattere sostanzialmente didattico, nella trattazione lella meccanica l'Aurar ebbe per consuetudine d'intrattenere i vari Congressi ai quali intervenne di persona.

Per bontà di esposizione più che per novità di risultati meritano lode alcuni lavori del Padula (Napoli, 1845) sulle equazioni per il moto de'li- 
12 Cerruti: To matematiche pmre e miste nei primi dodici Congressi'

quidi, sui solidi di egual resistenza, sulla flessione delle travi incastrate ai due estremi, e sull'equilibrio dei müri che sostengono la spinta delle terre; ed al medesimo titolo va segnalata una monografia del professore Овис (Tоrino, 1840), pubblicata poi in appendice al trattato di fisica generale del Mozzon $\left({ }^{31}\right)$, sulla determinazione grafica delle leggi del moto cagionato nei corpi dalle percosse.

Ma di maggior momento apparirono senza dubbio. alcuni tentativi del Condzza (Milano, 1844; Genova, 1846) per una teoria del calore fondata sulla dottrina delle ondulazioni, che potrebbero servire di propedeutica ad un modo di analisi degli effetti termo-meccanici $\left({ }^{32}\right)$.

Degne pure che se ne faccia menzione sono varie notizie riguardanti argomenti di astronomia e di meccanica celeste, tra le quali mi contenterò di ricordare quelle del CaRLin relative alla determinazione delle costanti dell'orbita lunare ed agli elementi parabolici della cometa scoperta a Roma il 23 agosto 1844 (Vilano, 1844), del Santini intorno alle perturbazioni prodotte da Giove e da Saturno sul moto della cometa di Biela (Padova, 1842), e finalmente di Brela sulla relazione fra i movimenti progressivi de' corpi celesti secondari col moto rotatorio del rispettivo corpo primario (Padova, 1842).

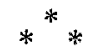

Mi parrebbe di venir meno al debito mio se terminassi questa rapida colsa senza accennare alla presentazione fatta da Carbo Barbage al Congresso di Torino (1840) della sua ingegnosa e celebre macchina calcolatrice, sulla quale il Menabres compì uno studio particolareggiato $\left({ }^{33}\right)$; studio che eble al suo tempo molto grido e, stampato dapprima nella Bibliothèque universelle di Ginevra, fin quasi subito riprodotto, voltato in inglese, e commentato, in una collezione di Memorie scientifiche pubblicate a Londra per cura di R. TAYLOR.

Per il medesimo motivo non posso tacele dei vari apparecchi sottoposti da Trto Gonnelua al giudizio del Congresso di Firenze (1841) tra i quali per il matematico ha particolare interesse un planimetro per la quadratura lelle aree piane, che fu come l'origine dei vari strumenti integratori ideati in seguito. L'apparecchio del Gonnella, al quale l'autore dedicò una Memoria illustratrice pregevolissima per sè $\left({ }^{34}\right)$, forniva l'area racchiusa tra una. curva, una retta e due peipendicolari a quest'ultima e corrispondeva alla ordinaria scomposizione di un'area piana in trapezi infinitesimi con rette perpendicolari all'asse delle ascisse. 


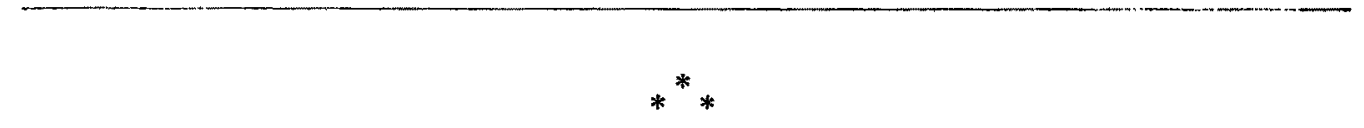

Ho già detto che nelle tre sessioni di Siena (1862), Roma (1873) e Palermo (1875) le matematiche furono poco o nulle rappresentate se non forse per studi, che vi hanno un nesso molto indiretto. Tali, per esempio, dovranno dirsi le lunghe e minuziose determinazioni (Roma, 1873) sperimentali del colonnello Pretro Contr intorno alla resistenza d'attrito nei corpi solidi, allo scopo principale di riconoscere l'influenza della velocità su tale resistenza, determinazioni che furono poi riunite in una voluminosa Memoria dell'Accademia dei Lincei $\left({ }^{35}\right)$; e così pure gli studi sperimentali del medesimo colonnello sulla resistenza dei materiali, di cui l'Accademia dei Lincei accolse nei suoi Atti la parte relativa alla flessione della pietra serena $\left({ }^{36}\right)$.

Lo stesso carattere hanno ancora gli accuratissimi e svariati lavori del Pısatr (Palermo, 1875) sull'elasticità dei metalli a diverse temperature $\left({ }^{37}\right)$. Nell'esecuzione dei quali lavori il Prsatr acquistò un'abilità sperimentale veramente rara, che lo mise in grado di condurre poi a splendido termine l'impresa, assunta insieme col Pucu, della determinazione del valore assoluto della gravità in Roma e dell'attrito interno di varie specie di gas $\left({ }^{38}\right)$.

Per certi lati confina similmente colla matematica applicata la Memoria letta dal Gerboñ nel Congresso di Roma (1873) dove per la prima volta con plastica evidenza e somma precisione sono posti i fondamenti razionali della contabilità e data forma e contenuto scientifico a una dottrina che era sempre rimasta nel dominio dell'empirismo $\left({ }^{39}\right)$.

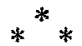

Onorevoli colleghi - Sebbene io mi sia ingegnato di riassumere imparxialmente e con ogni migliore diligenza l'opera matematica dei passati Congressi, non ho la pretesa di essere stato completo: può essere che qualche lavoro di pregio mi sia inavvertentemente sfuggito e che di qualche altro abbia taciuto per non averlo apprezzato al suo giusto valore. $\dot{\mathrm{E}}$ un difetto del quale non mi voglio scusare; ma lo schivarlo sarebbe tornato difficile, perchè è sempre malagevole sottrarci all'influenza delle inclinazioni e delle opinioni che si radicano in ognuno di noi come conseguenza degli studi personali. Sarei contento per altro se colla mia stringata rassegna fossi almeno riuscito a mostrare infondato il troppo sfavorevole giudizio che suol portarsi dello stato delle scienze matematiche nel nostro paese nei primi 50 anni del secolo passato.
\end{abstract}


$\mathrm{Fu}$ deplorato che in Italia non si fosse mai potuta costituire una tradizione matematica, o che fosse mancata la continuità anche là dove momentaneamente qualche ingegno eletto aveva saputo suscitare l'amore all'investigazione geometrica. Il fatto, vero in certa misura, ebbe varie cause, che furono dette e ripetute tante volte: ma tra esse ve n'ha una molto umile, che forse ha esercitato e può esercitare un influsso più grande di quello che ordinariamente gli si suole attribuire. In un paese povero, dove il movimento e la trasformazione della ricchezza si riducono a proporzioni minime, dove i grandi problemi dai quali dipendono la floridezza e la potenza dello Stato, non sono affrontati, l'utilità pratica della matematica non va al di là degli elementi indispensabili per la vita comune: e l'interesse per le quistioni più elevate e riposte non può essere che retaggio casuale di qualche mente privilegiata. Tale era la condizione dell'tralia nella prima metà del secolo xix. Ma oggi non è più cosi. Il merariglioso risveglio industriale; le opere colossali per la sistemazione e l'esercizio delle comunicazioni aeree, terrestri e marittime; il generale rinnovamento edilizio; lo sfruttamento dell' energie naturali in tutte le loro forme; l'assetto dei grandi servizi di Stato; la costituzione di nuovi istituti economici imposti dall'incessante trasformazione sociale, hanno richiesto già e richiedono senza tregua la risoluzione di problemi complessi, pei quali l'ausilio della matematica nelle sue parti più delicate è così necessario e prezioso, che sono costretti ad acquistarvi famigliarità moltissimi, a cui la scienza non è un fine ma un mezzo. Queste necessità di ordine tecnico esercitano per converso un benefico influs:o sui cultori della scienza per sè, tanto rispetto all'indirizzo delle loro speculazioni, quanto rispetto al perfezionamento dei metodi di ricerca. Poichè la matematica astratta nella nuova vita italiana rappresenta non più una dottrina di lusso e decorativa, ma un valutabile fattore economico, è lecito sperare che il cammino ascendente, il quale dura con tanta fortuna da oltre mezzo secolo, non abbia ad allentarsi, ma continui con vigore moltiplicato. A raggiungere tale intento riuscirà efficacissima l'azione della nostra Societì accomunando ed organizzando studi e studiosi. Essa nei primordi si valse (lella scienza per cooperare alla formazione di una coscienza politica nazionale. Ora ha avanti a sè un ideale non meno nobile e più proprio alla sua indole; quella di creare una coscienza scientifica nazionale. 


\section{NOT E}

De'vari autori sono citate, oltre le pubblicazioni alle quali si fa allusione espressa nel testo, anche alcune altre che hanno con esse una stretta comessione.

(') Paol (Pietro). Elementi di Algebra. Pisa, Tip. della Soc. Letteraria, MDCGCIII-MDCCCIV, 1, pref., pp. $r-\mathrm{VII}$.

(2) V. ex. g Betrnam (Eugenio) in Collectanen mathematicr nunc primum edita. Milano, Ulrico Hoepli, 1881, pp. I-III.

(') Manmstwa (Carlo Giovanni). Note sur la converyence des séries. Upsala, Nova Acta Soc. Se., XII, 1844, pp. $255-270$.

- Teber einen Satz von fler Convergenz her Reilen. Grunert, Archiv, VI, 1845, pu. 38-41.

Si tratta del teorema: «Se is $\sum_{t}^{\infty} u_{n,}$ una serie a termini positivi divergente con $\lim _{n=\infty} u_{n}=-=0$,

sono convergenti le due serie $\sum_{n}^{\infty} u_{n} \cos (\omega+n \theta), \sum_{0}^{\infty} u_{n} \operatorname{sen}(\omega+n \theta)$, traune che per $\theta$ multiplo della circonferenza ».

(4) Hommaren (Hjalmar). Sur la convergence les séries trigononnétriques procédant suivant les multiples d'un même arc. Liouville, Journ. d. math. pures et appliquées, XVI, 1851, pp. $186-190$.

(5) Per notizie minute sui lavori scientifici del Cinò e sulle controversie derivate dalle sue ricerche intorno alla serie di Lagrange vedi:

Genoccur (Angelo). Notizie intorno alla vita ed agli soritti di Felrae Chò. Bullettino di Bibliografia e di Storia delle Scienze matematiche e fisiche pubblicato da B. Boncompagni, 1871, IV, pp. 363-380.

Boncompagnt (Baldassarre). Catalogo dei lavori di Felice Chiò. Id., id., pp. 381-400.

Ferraris (Galileo) in Discorsi per l'inaugurazione del busto di Fndice Gerò nella $r$. Universitç di Torino al 28 novembre 1872. 'Torino, Stabilimento clell'Unione tipografico-ediLrice, 1874, pp. 5-28. Il discorso del Ferraris fu riprodotto nella raccolta delle sue Opere pubblicate per cura dell Associazione elettrotecnica italiana. Milano, Ulrico Hoepli, 1909-904, III, pl. 331-35L.

Marie (Massimiliano). Théorie tes fonctions ale variables imaginaires. Paris, GauthierVillars, 1874-76, III, pl. 280-313.

$\left(^{6}\right)$ I due lavori principali del MexdBRea sulla serie di Lagrange hamno per titolo:

Mémoire sur la série de latgrange. [1843]. Torino, Acc. Sc., Memorie, 2.a serie, VIIl, 1846, pp. 91-128; 
Observations sur la veritable interprétation de la série de Lagrange. [1846]. Torino, id., id., $X, 1849$, pp. 111:-162.

Insieme con essi giova altresì ricortare la relazione del medesimo Menaprfa sopra una Memoria del Chiò intorno alla convergenza e le proprietà della serie di Lagrange, letta nella seduta del 2 luglio 1843 della R. Accademia delle Scienze di 'Torino, e stampata, coll'aggriunta di tre Note, nell" « Antologia italiana, Giomale di Scienze, Lettere od Arti». Torino, a11. I, t. II, 1847, pp. 65-88.

(7) Gli articoli di polemica cui si accenna nel testo sono:

Ghocchr (Angelo). Intomo ad wat lettera del sig. comte L. F. Mnanmena. Bullettino di Bibliografia ecc., ece., V, 1872, pp. 535-549.

- Richiamn a favore ai Frutan Chò. Bullettino di Bibliogratia ece, ece. VI, 1873, pp. $153-158$.

- Su d'una controversia intomo alla serie ri Lagrange. Torino, Ace. Sc., Atti, VIIL, 1872-73, pp. 18-31.

- Breve risposta al signor conte L. F. Menasrka. Bullettino di Bibliografia ece, oce, VI, 1873 , pp. 530-532.

- Observations relatives à une Note de M. MÉnABŔa concernant la série le Lagrange. Paris, Ac. des Sc., Comptes-rendus, LXXVII, 1873, pp. 1541-1544.

Menabrea. (Luigi Federico). Intomo ad uno scritto del sig. prof. Angalo Ganocchi. Bullettino di Bibliografia ece., ecc., V, 1872, pp. 301-305.

- Un'ultima lettera sulle peripezie della serie di Lagrange in risposta al prof. AnGuLo Grinocchi. Bullettino di Bibliografia ecc., eee, VI, 1873, pp. 435-457.

- Note sur l'identité des formules données par Cauchy pour déterniner les conditions de convergence te la série de Lagrange avec celles qui ont été établies par Lagrange lui-mêne. Paris, Acad. des Sc., Comptes-rendus, LXXVII, 1873, pp. 1358-1361. - Les Mondes, XXXII, 1878 , pp. 673-675.

(8) Minıch (Seralino Raffaele). Sugli integrali algebrici d'un sistema di equazioni differenziali $i$ cui termini sono integrabili per mezzo di trascendenti abeliane e sulla proprieta fondamentale di simili trascendenti. Venezia, Memorie dell'i. r. Istituto Veneto di Sc., Lett. ed Arti, III, 1847, pp. 269-328.

(b) Genocch (Angelo). Sopra una costruzione del teorema di Abel. Annali di Matematica pura ed applicata, I, 1858, pp. 33-40.

Briosan (Francesco). Sur l'intégration des équations ultra-elliptiques. Crelle, Jouru. f. die reine u. angew. Math., LV, pp. 55-60; - od anche $t$. V delle sue Opere matematiche, attualmente in corso di stampa, pp. $287-291$.

(10) Trud (Nicola). Delle relazioni tra i detesminanti di due sezioni coniche, l'una iscritta, l'altra circoscritta ad un potigono irregolare. Napoli, Acc. Sc., Rendiconto, II, 1843, pp. 89-93.

- Rappresentazione geometrica immediata dell'equazione fondamentale nella teoria delle funzioni ellittiche con diverse applicazioni. Napoli, Acc. Sc., Memorie, 1, 1852-54, pp. 63-100.

- Ricerche risguardanti la moltiplicazione ed adlizione geometrica delle funzioni ellitiche. Napoli, Acc. Sc., Rendiconto, II, 1853, pp. 66-69.

- Studii intorno ad una singolare eliminazione, con applicazione alla ricerca della relizione tra gli elementi di due coniche, l'una iscritta, l'altra circoscritta al un poligono, ed ai conrispondenti teoremi del Poncelet. Napoli, Ace. Sc., Rendiconto, I, 1862, pp. 198-210:

id., id. Atti, I, $1863, n^{\circ} 6$. 
- Sui teoremi del Poncelet relativi ai poligoni iscritti e circoscritti alle coniche. Gionale Ii Mat. ad uso degli studenti delle Univ, italiane, I, 1863, pp. 81-90, 125-126.

(11) Fristant (Paolo). Analisi di alcune equnzioni trascendenti. Effemeridi astronomiche Ui Milano per l'anno 1845, Milano, 1844, Appendice, pp. 3-12\%.

- Sull'integrazione delle equazioni differenziali ordinarie di $1.0^{\circ}$ ordine e lineari per un numero qualunque di variabili. Effemeridi astronomiche di Milano pel J'anno 1848, Milano, 1817, Appendice, pp. 3-146.

(12) Lavada (Giovanni Maria). Sulla integrazione dell'equazioni non lineari fi natura qualunque alle derivate parziali del prim'ordine fra qualsivoglia mumero di cariabili. Ciatania, Ace. Gioenia, Atti, VII, 1850, pp. 17-81.

${ }^{\left({ }^{18}\right)}$ TARDY (Placido). Sui differenziali a indice qualunque. Annali di matematica pura ed applicata, I, 1858, pp. 135-148.

${ }^{\left({ }^{14}\right)}$ - Sopra alcuni punti della teoria del moto dei liquirli. Firenze, Gio. Mazzoni, 1847, pp. $1-29$.

(15) Gnocch! (Angelo). Di una Nota del banone Prana. Casi particolari del moto dei liquili. Annali di matematica pura ed applicata, I, 1858, pj. 383-396. Delle medesime quistioni, senza per altro riferirsi agli studî del TAndx, s'era occupato jl Genocchi in altro lavoro prorcedente dal titolo: Sopra una formula di Tagrange spettante al moto de'liquidi ne' vasi in: Amali di Scienze matematiche e fisiche, VIIl, 1857, pp. 396-420.

(16) La comunicazione del BoRcraRDT fu riprodotta integralmente nella collezione dells: sue Opere, Berlin, G. Reimer, 1888, pp. 465-466. Essa si aggira sul medesimo soggetto della dissertazione presentata nel 1843 dal Borchardt per il dottorato alla Facolta filosofica di Königsberg, della qual dissertazione non si potè trovare l'originale e si conosce il solo titolo: De integratione quarundam aequationum differentialium una cun varis arl theorian. integralium ellipticonum applicationibus.

(17) JAGOB (Carlo Gustavo Jacob). Sopra le funzioni di Laplace, che risultrmo tallo sviluppo dell'espressione

$$
\left(a^{2}-2 a a^{\prime}\left[\cos \omega \cos \varphi+\operatorname{sen} \omega \operatorname{sen} \varphi \cos \left(\vartheta-\vartheta^{\prime}\right)\right]+a^{\prime 2}\right)^{-\frac{1}{2}} .
$$

Giomale Arcadico, XCVIII, 1844, pp. 59-66.

- Sulla condizione di uguaglianaa di due radici dell'equazione cubica, dalla quale rijentono gli assi principali di una superficie del second'ordine. Id., XCIX, 1844, pp. 3-11.

- Sul principio dell'ultimo moltiplicatore, e suo uso come auovo principio generale di meccanicu. Id., XGIX, 1844, pp. 129-146.

Oltre le comunicazioni qui ricordate, il Gionnale Arcadico contiene ancora, tradotti in

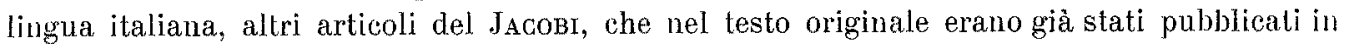
riviste scientifiche straniere. La notizia degli anti, che nella composizione de' suoi lavori in lingua italiana, ebbe il JAcoBr dal Chelin, la appresi dalla viva voce di quest'ultino.

${ }^{\left({ }^{8}\right)}$ Stminfr (Jacopo). Teoremi relativi alle coniche iscritte e circoscritte. Giomale Arcadico. XCIX, 1844, pp. 147-161.

- Del baricentro di curvatura delle curve piane. Id., CI, 1844, pp. 1-31, 121-160. ì una traduzione, eseguita per cura del Dr. Luigi Schlaefli, dall'originale terlesco di una Memoria

Annali di Matematica, Serie.III, Tomo XV. 
letta il 15 aprile 1838 all'Aceademia delle Scienze di Berlino e poi stampata nel Giornale di Crelle, XXr, 1840, pp. 33-63, 101-133.

( $\left.{ }^{19}\right)$ Panura (Fortmato). Ricerche di analisi rpplicata alla geometria. Napoli, Ace. Sc., Rendiconti, III, 1844, pp. 241-256, 321-328, 401-408; IV, pp. 15-82.

- Delle curve di quarto grado che hanno tre punti di regresso di prima specie. Napoli, Ace. Sc., Rendiconti, I, 1852, pp. 29-34; il., id., Memorie, I, 1852-54, pp. 31-48; - Roma, Imnali di Scienze matematiche e fisiche, III, 1852, pp. 383-387.

- De' punti multipli delle curve algebriche, Annali di Scienze matematiche e fisiche, III, 1852, pp. $911-231$.

$\left.{ }^{20}\right)$ Mossort (Ottaviano Fabrizio). Sur les forces qui régissent la constitution intériente tes corps. Apercu pour servir it la determination de la canse et les loix de l'action molecutlaire. Turin, Imprimërie loyale, MDCCGXXXVI, pp. 1-34: - voltata nell'idioma inglese in Taylos, Scient. Mem., I, 1837, pp. 448-469: - riprodotta nelloriginale francese in

ZöLL.Nhr (Federico). Erklarung der miversellen Growitution rus den statischen Wirkungen. der Elektricitöt unl die allgemeine Bedextung des Weber'schen Gesetzes. Leipzig, L. Staackmann, 18:2, pp. 83-119.

(21) - Tezioni elementari ni Fisica matemntica. Firenze, G. Piatti, 1843-45; 2 voll. II primo volume comincia con un discotso, dove i liprodotta la sostanza della Memoria di cui alla nota precedente.

$\left.{ }^{29}\right)$ - Sul principio che la riflessione e la rifrazione su di una superficie univifrangente polwizzano nelle due porani in cui vien hiviso il raggio incidente due quantitro di luce uguali, rispettivanente in a piani ortogonali fra lom. Gionale toscano di Se. med., fis. c nat., I, 1810, pp. 330-337.

- Sulla causa della dispersione della luce nel sistema delle ondulazioni. Id., I, 1840, pp. $337-341$.

- Dell'azione delle forze molecolari nella produzione de' fenomeni di capillaritì. Bibl. it., XCTIT, 1840, pp. 63-80; - Nuovi Ann. Sc. nat., IT, 1840, 1). 390-419; - Taylor, Scient. Mem., III, 1843, pp. 564-577.

- Nota sopra un fenomeno capillare osservato dal Dr. Young. Bibl. it., XCVIII, 1840, pp. 365-375; - Taylor, Scient. Men., 1II, 1843, pp. 578.586.

- 1) Riflessioni intorno alla forza epipolica. 2) Deduzione delle formole della doppia rifrazione di FRESEL dalle equazioni generali tel movimento dell'etere disseminato noi cmpi cristallizati. Pisa, II Cimento, 1I, 1844, pp. 429-437.

- Sulle proprietì degli spettri di Fraunhofer formati dai reticoli, ed analisi della luce che somministrano. Pisa, Ann. Univ. Toseane, Sc. cosmologiche, I, 1846, pp. 181-204: 'Taylor, Scient. Mem., V, 1852, pp. 435-452.

- Commicazioni sopra alcuni argomenti di acustica e ti ottica fisica. Pisa, Il Cimento, IV, 18:6, pp. 97-106.

- Considerazioni sulle forze di capillarità e coesione dei liquidi relative alle recenti esperienze dei sigg. Henry, Donny ed Hager. Pisa, II Cimento, IV, 1846, pp. 439-456.

- Discussione analitica sull'infuenza che l'azione di un nezzo dietettrico ha sulla distribuzione dell'elettricitc̀ alla superficie di piì corpi elettrici disseminati in esso. [1846]. Modena, Soc. it. delle Sc. (detta de' XL), Memorie, XXIV, 1850, pp. 49-74.

- Azione de' parafulmint. Il Nuovo Cimento, XVI, 1862, pp. 74-79. 
(28) Plana (Giovanni). Sur la distribution de l'électricitè à la swrface de deux sphères conructrices complètement isolées. Torino, Acc. Sc., Memorie, 2. ${ }^{\text {a }}$ serie, VII, 1845, pp. 71-401.

- Sur la distribution de l'électricité à la surface intérieure et sphérique d'une sphère creuse de métal et à la surface d'une antre sphère conductrice électrisée que l'on tient isolée dans sa cavité. [1854]. Toriuo, Acc. Se., Memorie, 2.a serie, XVI, 1857, pp. 57-96.

(4) Darboux (Gastone). Sur dewx Mémoires de Potsson relatifs à la distribution de l'électricité. Bulletin des Sciences mathématiques, 2.òme série, XXXI, 1907, pp. 17-28.

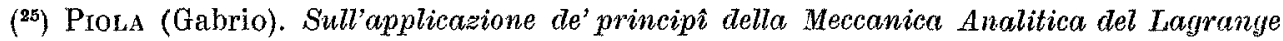
ai principali problemi. Milano, Regia Stamperia, 1825, pp. I-XXIII, 1-252.

- Trattato sul calcolo degli integrali definiti. Milano, Opusc. mat. e fis., I, 1832, pp. 73-96, 169-200, 273-296, 345-375; - II, 1834, pp. 105-132, 345-372.

- La Meccanica de' corpi naturalmente estesi trattata col Calcolo delle variazioni. Opusc. mat. e fis., I, 1832, pp. $201-236$.

- Sui principî e sugli usi del Calcolo dé residui (da varie Memorie del sig. A. L. CaUchy). Milano, Opusc. mat. e fis., II, 1834, pp. 237-260.

- Nuòva analisi per tutte le questioni della meccanica molecolare. Modena, Soc. it. delle Sc. (detta de' XL), XXI, 1836, pp. 155-329.

- Intorno alle equazioni fondamentali del movimento dei corpi quali si vogliono, consilerati secondo la naturale loro forma e costituaione. [18z5]. Modena, Soc. it. delle Sc. (detta (te' XL), XXIV, 1848, pp. 1-186.

Piola (Gabrio) e Frisiani (Paolo). Sulla meccanica celeste e sopra mo nuovo calcolo chiamato « Calcolo dei limiti». (Memoria di A. L. CAuchy tradotta ed annotata). Milano, Opuse. mat. e fis., II, 1834, pp. 1-84, 133-202, 261-316.

(26) Brioschi (Francesco). Prefazione ad ma Memoria postuma di G. Prola dal titolo: «Ulteriori considerazioni sul moto dell'acqua in vasi, canali e fumi». Milano, Memorie dell'i. r. Istituto Lombardo di Sc, Lett. ed Arti, L. a Serie, III, 1852, pp. 283-298; -- od anche 1. III delle sue Opere matematiche, pp. 119-135.

${ }^{(27)}$ Venturoli (Giuseppe). Sull'efflusso dell'acqua dai vasi conici in : Ricerche geometriche ed idrometriche fatte nella Scuola degli ingegneri pontifici d'acque è strade l'anno 182 4 . MîImo, P. E. Giusti, MDCCCXXII, pp. 1-11. - Il Venturoli fin dallanno 1810 in un'appendice alla seconda edizione degli Elementi di Meccanica ed laraulica aveva trattato in generale il problema del moto di un velo fluido piano, nelle solite condizioni restrittive ricordate nel discorso, ed applicata la teoria generale al caso delle pareti rettilinee.

(28) Groulo (Carlo Ignazio). Di un caso particolare della dottrina dell'efflusso dell'acqua a $a^{\prime}$ vasi. Torino, Stamperia reale, 1839 , pp. 1-38.

${ }^{(29)}$ B ETT1 (Enrico). Sopra la determinazione analitica dell'eftusso dei liquicli per una piccolissima apertwa. Annali di Sc. mat. e fis., I, 1850, pp. 425-443; - od anche t. I delle sur Opere matematiche, pubblicate per cura della R. Accademia de' Lincei, Roma, 1903, pp. 3-16.

${ }^{\left({ }^{\circ o}\right)}$ Bidone (Giorgio). Sur la deitermination thérique de la section contractée des veines liquitles. [1829]. 'Torino, Ace. Se., Memorie, XXXIV, 1830, pp. 363-387.

( $\left.{ }^{81}\right)$ Obicr (Pietro). Le leggi del moto cagionato ne' corpi dalla percossa grafieamente determinate in: Mozzoni (Audrea), Elementi di Fisica generale, 7. ${ }^{\text {a }}$ edizione, 1. fiorentina, Firenze, G. o. S. Jouhaud, 1841, Appendice, pp. 967-324.

(32) Codazza (Giovanni). Sulla teoria della propagazione dellt luce omogenea nei mezzi omogenei. Milano, Soc. tip. dei Classici jlaliani, 1840, P1. I-XII, 1-104. 
20 Cerruti: Le matematiche pure e miste nei primi dodlici Congressi, ecc.

- Esposizione dei principii generali sull'equilibrio e sul moto delletere nell'intemo dei conp $^{i}$ pesanti. Majocchi, Annali di Fis., Chim. e Mat., Milano, 1844, XVI, pp. 252-257.

- Sulle induzioni molecolari prodotte dalle ondulazioni dell'etere. Milano, Giornale dell'i. 1 . Istituto Lombardo di Sc., Lett. ed Arti, nuova serie, [V, 1852, pp. 199-230.

- Sulla polarizzazione rotatoria della luce sotto linfuenza delle azioni elettromagnetiche. Milano, Giornale dell'i. r. Istituto Lombardo di Sc, Lett. ed Arti, nuova serie, IV, 1852. pl. $491-538 ; \mathrm{V}, 1853$, pp. $299-342$.

- Considerazioni sulla possibilitì dell'esistenza di un mezzo magnetico negli spazi vuoti ri materia ponderabile. Milano, Giornale dell'i. r. Istituto Lombardo di Sc., Lettere ed Arti, muova serie, VIJI, 1856, pp. 247-261.

("s) Menarrea (Luigi Federico). Notions sur la machine analytique de M. Charles BA\}13AG. Bibl. universelle; XLI, Genève, 1842, p1. 353-377; - Taylor, Scient. Mem. III, 1843, p). $666-731$.

(34) Gonned.LA (Tito). Opuscoli matematici. Descrizione di una macchina per quadraie le figure piane. Firenze, Gio. Mazzoni, 1841, pp. 135-194, con due tavole.

${ }^{\left({ }^{5}\right)}$ Coxt (Pietro). Sulla resistenza di attrito. Roma, R. Acc. de' Lincei, Atti, 2." Serie, II, 1875, pp. 16-200, con 25 tavole.

${ }^{\left({ }^{6}\right)}$ - Sulla resisteba alla Ressione della pietra «serena». Roma, R. Ace. de'Lincei, Atti, 2." Serie, II, 1875, pp. 408-416, con una tavola.

(37) PISATr (Giuseppe). Sulla tenacita del ferm a diverse temperature. Firenze, Soc. it. delle Se. (detta de' XL), Memorie, 3.a serie, II, 1869-76, pp. 321-342, con una tavola.

- Sull'elasticitci de' metclli a diverse temperature. Palermo, Gazzetta chimica italiana, VI, 1876, pp. 235-32, 57-88, 176-196; VII, 1877, pp. 61-89, 173-188, con tre tavole.

- Ricerche sperimentali sulla tenaciti de' metalli a diverse temperature. Roma, R. Acc. de' Lincei, Memorie, 3." serie; I, 1877, pp. 179-194, con una tavola.

$\left({ }^{38}\right)$ Pisati (Giuseppe) e Puaci (Enrico). Sulla lungheza del pendolo a secondi. Roma. R. Ace. de' Lincei, Memorie, 3." serie, XV, 1885, P1. 57-231, con quattro tavole.

- Sulla linghezza del pendolo semplice a secondi in Roma. Esperienze eseguite dai professoni G. PISATl ed F. Puccl, pubblicate per cura di Vincenzo Reina. Roma, R. Ace. dei Lincei, Memorie, 5." serie, I, 1894, pp. 3-162, con una tavola.

${ }^{39}$ ) Carbonr (Giuseppe). Sullordimanento della contabiliti dello Stato. Firenze, Giuliani, 1866, Hp. L-60, con una tavola.

- Prini saggi di logismografia presentati all'XI Congresso legli Scienziati italiani. Firenze, Tip. La Minerva, 1875, pp. 1-8.

- Sur l'importance d'unifier les études de la comptabilité. Roma, Eredi Botta, 1887. lip. 1-292.2. 